\title{
Sigma-1 and N-Methyl-D-Aspartate Receptors: A Partnership with Beneficial Outcomes
}

\author{
Mohan Pabba ${ }^{\mathrm{a}}$ Etienne Sibille $\mathrm{a}^{\mathrm{a}-\mathrm{c}}$ \\ ${ }^{a}$ Neurobiology of Depression and Aging, Campbell Family Mental Health Research Institute, Centre for Addiction \\ and Mental Health, and Departments of ${ }^{\mathrm{b}}$ Psychiatry and ${ }^{\mathrm{C}}$ Pharmacology and Toxicology, University of Toronto, \\ Toronto, Ont., Canada
}

\section{Key Words}

Sigma-1 receptor NMDA receptors $\cdot$ Schizophrenia .

Neuroprotection

\begin{abstract}
Sigma-1 receptors ( $\sigma-1 R$ ) are interorganelle signaling molecules, which have been implicated in synaptic plasticity, primarily by enhancing the function of $\mathrm{N}$-methyl-D-aspartate receptors (NMDARs). On the other hand, excessive influx of calcium via activated NMDAR can cause excitotoxicity. Yet, despite their NMDAR-enhancing role, multiple lines of evidence suggest that $\sigma-1 R s$ are involved in neuroprotection. The mechanism underlying these intriguing opposing effects is not known. Recent studies now suggest the possibility that $\sigma-1$ Rs could exert neuroprotective effects via targeted disruption of protein-protein interactions between NMDARs and their associated intracellular signaling machinery, specifically the neuronal nitric oxide synthase (nNOS). This targeted disruption of protein-protein interactions between NMDARs and nNOS results in lower levels of nitric oxide generation, thus having a neuroprotective effect. Here, we briefly summarize aspects of $\sigma$-1R-mediated enhancement of NMDAR function and possible neuroprotection. Indepth mechanistic understanding of $\sigma-1 R$ modulation of
\end{abstract}

NMDAR function, which preserves $\mathrm{Ca}^{2+}$ homoeostasis while limiting excitotoxicity would provide valuable information for designing novel as well as improving prevailing therapeutic strategies.

(c) 2015 S. Karger AG, Basel

A remarkable number of intra- and extracellular components in a highly organized and intricate manner mediate neurons' health and spatiotemporal communication. Among many components, calcium ion $\left(\mathrm{Ca}^{2+}\right)$ has a prominent role and, in fact, stands at the crossroads of having either beneficial (e.g., synaptic plasticity) or toxic (e.g., excitotoxicity) effects on the neuron [1-3]. An optimal and regulated intracellular influx of $\mathrm{Ca}^{2+}$ into the neuron elicits signaling cascades that strengthen the communication between synapses (i.e., underlying synaptic plasticity) [3]. However, an excess influx of $\mathrm{Ca}^{2+}$ can also elicit signaling cascades, which in this case may result in a toxic insult to neurons and glia [1]. While the overarching mechanisms underlying the dichotomous effects of $\mathrm{Ca}^{2+}$ are not fully understood, several seminal discoveries have demonstrated a predominant role for N-methyl-Daspartate receptors (NMDARs), more specifically the NMDAR-associated intracellular signaling machinery, in

\section{KARGER 125}

C 2015 S. Karger AG, Basel

2296-9209/15/0011-0047\$39.50/0

E-Mail karger@karger.com

www.karger.com/mnp
Etienne Sibille

Centre for Addiction and Mental Health, University of Toronto

Room 134, 250 College street

Toronto, ON M5T1R8 (Canada)

E-Mail Etienne.Sibille@ camh.ca 
mediating the dichotomous effects of $\mathrm{Ca}^{2+}[4,5]$. Not surprisingly, neurons employ a number of specialized mechanisms to govern and tailor the function of NMDARs and/or the NMDAR-associated intracellular signaling machinery. These include mainly, but are not limited to, engaging modulators. The sigma-1 receptor $(\sigma-1 \mathrm{R})$ is one such modulator, which is known to enhance the function of NMDARs (i.e., heightened $\mathrm{Ca}^{2+}$ influx via NMDARs); however, $\sigma$-1Rs can also prevent the $\mathrm{Ca}^{2+}$-induced toxicity by modulating the function of a specific NMDAR-associated intracellular signaling component (neuronal nitric oxide synthase, nNOS), which generates toxic species (nitric oxide, NO). These intriguing opposing effects are discussed here. We provide a brief overview on NMDARs and $\sigma-1$ Rs and then summarize mechanistic aspects of $\sigma-1 \mathrm{R}$ modulatory action on the function of NMDARs and its association with nNOS.

NMDARs are glutamate-gated $\mathrm{Ca}^{2+}$-permeable ion channels [6]. They are heterotetrameric assemblies of two compulsory GluN1 subunits together with either two GluN2 (A-D) subunits or a combination of GluN2 or GluN3 (A and B) subunits. These glutamatergic receptors display a characteristic subunit- and age-dependent temporal and spatial distribution throughout the central nervous system. At the synapse, NMDARs exist as large macromolecular complexes that contain numerous types of molecules, including scaffold/signaling proteins, e.g., postsynaptic density-95 (PSD-95). The $\mathrm{Ca}^{2+}$ conductance through NMDARs at synapses acts as a regulator of excitatory synaptic transmission, and any sort of deregulation in NMDAR function leads to neurological disorders, including schizophrenia and stroke [7].

$\sigma-1$ Rs are intracellular proteins which primarily reside on membranes of the endoplasmic reticulum that are in juxtaposition to mitochondria [8]. They are also present on the plasma membrane and are ubiquitous in neuronal and non-neuronal cells. It is predicted that $\sigma$ - 1 Rs possess two transmembrane units along with short $\mathrm{N}$ - and long C-terminus tails. Recent investigations have disclosed $\sigma-1 \mathrm{R}$ as an interorganelle 'modulator' that has chaperonelike activity and also acts as an intracellular sensor in regulating $\mathrm{Ca}^{2+}$ homoeostasis $[8,9]$. Furthermore, $\sigma-1 \mathrm{Rs}$ have been implicated in several physiological functions, including shaping neuronal excitability and long-term potentiation, mainly through functional modulation of ion channels (e.g., NMDARs) [10].

Electrophysiology-based investigations demonstrated that activated $\sigma-1 \mathrm{R}$ enhances the frequency and amplitude of various NMDAR-mediated responses as well as NMDAR-dependent long-term potentiation [11-13].
Additionally, concurrent behaviour-based studies demonstrated improvements in learning and memory behaviour of animals after the activation of $\sigma$-1Rs that are experiencing NMDAR antagonism-induced amnesia [14, 15]. Two important features can be inferred from these multimodal studies: first, low doses of $\sigma-1 \mathrm{R}$ agonists enhance NMDAR function, while high doses $(\geq 10 \mu \mathrm{M})$ of $\sigma-1 R$ agonists do not enhance NMDAR function $[12,13]$. At the moment, it is unclear why high doses of $\sigma-1 \mathrm{R}$ agonists do not promote NMDAR function, but it is proposed that at high doses $\sigma-1 \mathrm{R}$ agonists can cross-react with NMDARs at their pore sites and eventually block ion channel conductance [16]. Second, the ameliorative effect on NMDAR function by $\sigma-1 \mathrm{R}$ agonists can be observed in minutes and sustained for hours [11]. This time scale window reflects the possibility that $\sigma$-1Rs may accomplish the functional enhancement of NMDARs through both direct and indirect (i.e., engagement of multiple cellular components) mechanisms (fig. 1). Indeed, $\sigma$-1Rs were shown to directly interact with GluN1 subunit of NMDARs [17], and this interaction may explain some of the facilitatory effects of $\sigma-1 \mathrm{R}$ agonists on NMDARs. On the other hand, electrophysiology-based examinations have revealed $\sigma$-1R-mediated recruitment of small-conductance $\mathrm{K}^{+}$-activated $\mathrm{Ca}^{2+}$ channels (SK channels) [11], $G$ proteins [18], and intracellular kinases (e.g., members of the Src family of kinases [19]) for increasing the function of NMDARs, but with the shortcomings that many aspects remain untested, such as, for instance, the effect of $\sigma-1 \mathrm{R}$ agonists on NMDAR subunit-dependent biophysical properties (e.g., gating, single-channel conductance, and open probability). This is especially important in lieu of changes in phosphorylation status - a posttranslational modification that influences the biophysical properties of an ion channel - of NMDAR GluN2B subunits following treatment with $\sigma-1 \mathrm{R}$ agonists [19]. Several other reports also demonstrate an increased phosphorylation of GluN1 subunits of NMDAR by kinases such as protein kinases A and C $[20,21]$ after ligand activation of $\sigma-1$ Rs. Furthermore, a recent biochemistrybased study showed $\sigma$-1R-mediated augmentation in expression, trafficking, and surface levels of NMDARs [22]. Equally, another recent report, although through a different viewpoint, demonstrated $\sigma$-1R-mediated suppression of NMDAR internalization and obstruction of NMDAR hypofunction [23]. An additional mechanistic modality likely to contribute is the indirect action of $\sigma-1 \mathrm{R}$ on NMDAR function through $\sigma-1 \mathrm{R}$-mediated $\mathrm{Ca}^{2+}$ mobilization from the internal stores [e.g., via inositol 1,4,5-triphosphate receptors (IP3Rs) on ER membranes] [9]. A
48

Mol Neuropsychiatry 2015;1:47-51 DOI: $10.1159 / 000376549$
Pabba/Sibille 
Fig. 1. $\sigma-1 \mathrm{R}$ enhancement of NMDAR function. $\sigma-1$ Rs adapt a multi-component approach to promote NMDAR function, including the inhibition of SK channels (a), $\mathrm{G}$ proteins (identity is unclear; $b$ ), and intracellular kinases (c) alongside with an increase in the expression, trafficking, and surface levels $(\mathrm{d})$ of NMDARs. In addition, $\sigma$-1R-mediated $\mathrm{Ca}^{2+}$ mobilization from ER via IP3 receptors may contribute to functional enhancement of NMDARs (e). The resultant increase in the influx of $\mathrm{Ca}^{2+}$ (blue arrow) further promotes synaptic plasticity $[9,11,18-22]$.

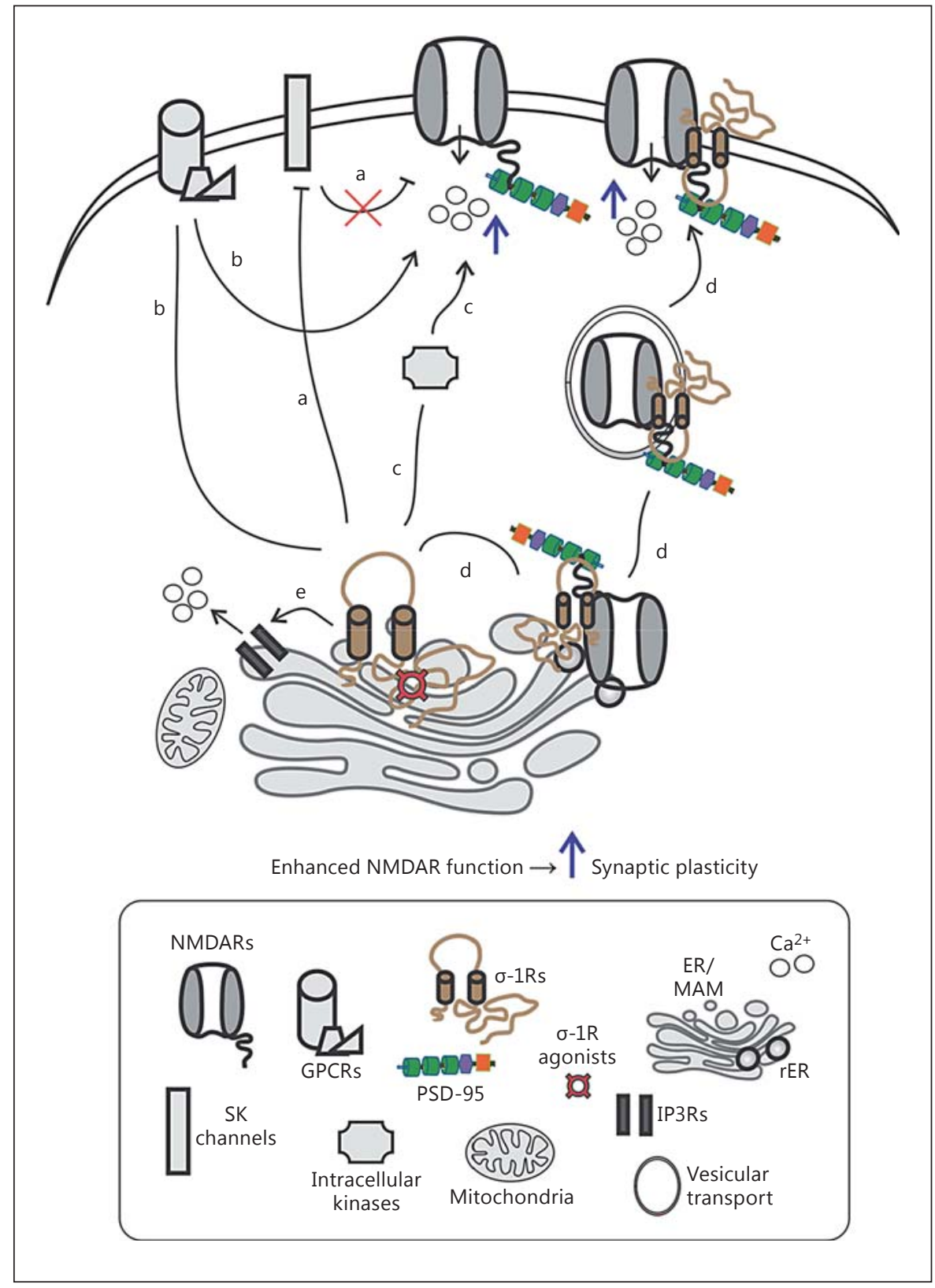

role for $\sigma$-1R-mediated $\mathrm{Ca}^{2+}$ mobilization in NMDAR functional augmentation seems plausible, but it remains to be directly tested. Early evidence in support of such a mechanism shows that activation of $\sigma$-1Rs in the presence of BAPTA [1,2-bis(o-aminophenoxy)ethane-N, $, N^{\prime}, \mathrm{N}^{\prime}$ tetraacetic acid], a $\mathrm{Ca}^{2+}$ chelating agent, does not induce a functional enhancement of NMDARs [11].

While $\sigma$-1R-mediated NMDAR functional enhancement seems semantic and beneficial as it relates to an improvement in the memory behaviour of animals $[14,15]$, it could also provoke an excitotoxic insult to the neuron. However, cell culture and behaviour examinations, based primarily on observations of (a) promotion and attenuation in the protein levels of survival and proapoptotic cellular components [24] and (b) reduction in the production of NO [25], suggest that $\sigma-1 \mathrm{R}$ ligands can act as neuroprotective agents. The reduction in the production of $\mathrm{NO}$ by $\sigma-1 \mathrm{R}$ ligands, if at all in doubt, can be due to the blockade of NMDARs by the ligands. However, this may not be the case. Rather, new findings hint that $\sigma-1$ Rs may 
Fig. 2. $\sigma-1 \mathrm{R}$-mediated neuroprotection. a In the absence of $\sigma-1 \mathrm{R}$ activation, the increased influx of $\mathrm{Ca}^{2+}$ through GluN2Bcontaining NMDARs can trigger neurotoxicity and neurodegeneration by increasing the activation of nNOS (eventually NO species) that is attached to PSD-95. b Recent investigations have demonstrated that the activation of $\sigma-1$ Rs leads to diminution of interactions between GluN2B subunits and PSD-95 as well as PSD-95 and nNOS (represented as a decrease in size). Reducing the interactions between proteins that can generate toxic species may be one of the underlying mechanisms of $\sigma$-1R-mediated neuroprotection via reduced neurotoxicity and neurodegeneration [22, 25-27].

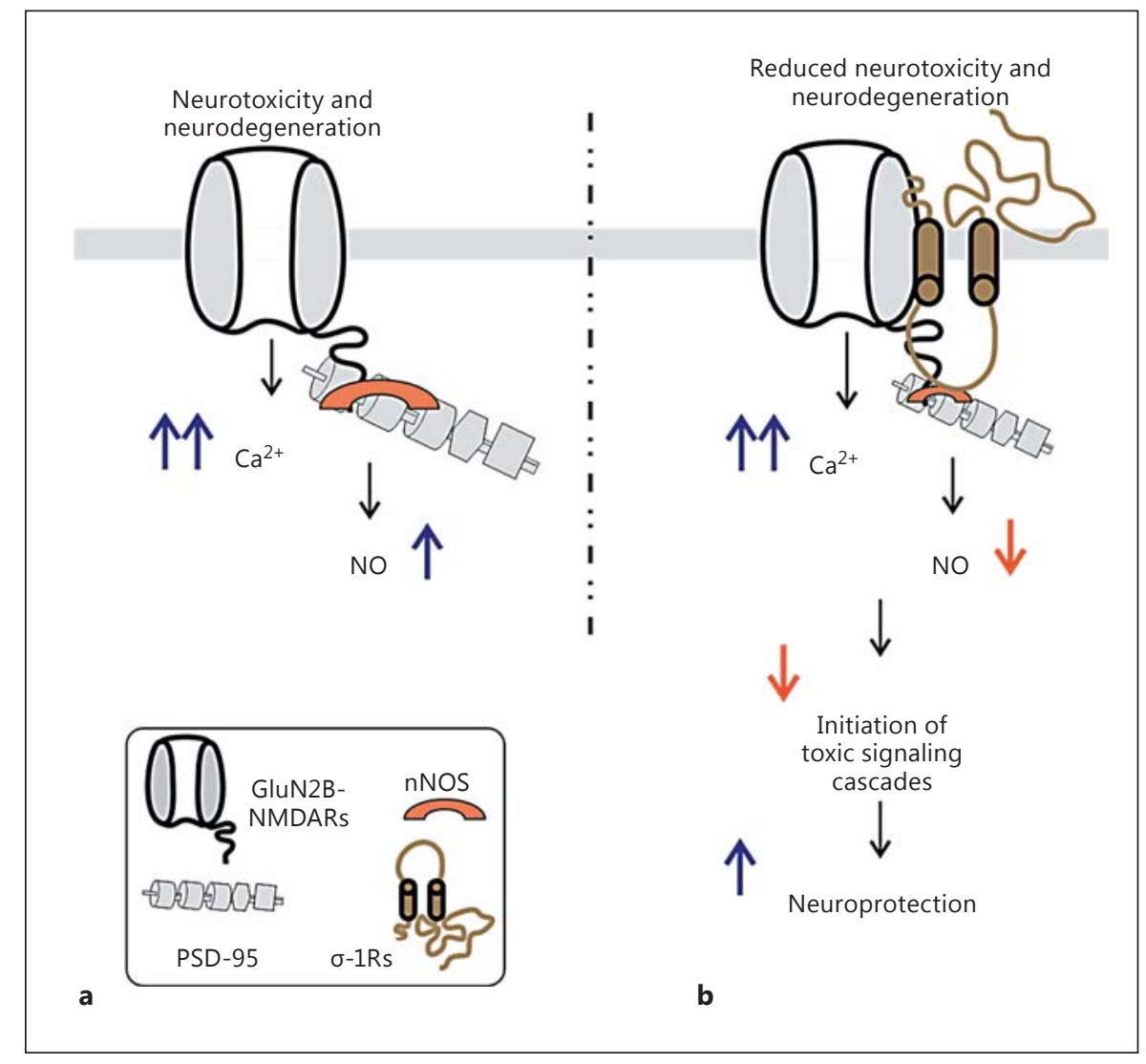

adopt an intriguing approach for wielding neuroprotection $[22,26]$, that is, $\sigma-1 \mathrm{R}$-driven attenuation of targeted protein-protein interactions that mediate neurotoxicity. It has been shown that excessive NMDAR-mediated $\mathrm{Ca}^{2+}$ recruits and activates nNOS that is attached to the NMDAR-PSD-95 complex. This tripartite complex is the driving force for neurotoxicity, and a disruption of these interactions between them greatly reduces toxicity-driven neuronal death [27]. Interestingly, agonist activation of $\sigma$-1Rs leads to decreased interactions between the NMDAR subunit (GluN2B) and PSD-95 [22] as well as PSD-95 and nNOS [26] (fig. 2), consequently giving rise to reduced neurotoxicity and neurodegeneration.

Why are these recent findings important? Neurons constantly have to recalculate and acclimatize to everchanging circumstances and perturbations. How such adaptation occurs is not well characterized, especially in cases of perturbation in neuronal $\mathrm{Ca}^{2+}$ homoeostasis (i.e., too much or too little NMDAR function and $\mathrm{Ca}^{2+}$ signaling [4]). Numerous investigations suggest a critical role for $\mathrm{Ca}^{2+}$ dyshomoeostasis in the pathogenesis of neuropsychiatric and neurodegenerative disorders [28]. On the other hand, modulation of NDMAR function is beneficial under many circumstances and a current target for therapeutic purposes. Thus, the fact that $\sigma-1 \mathrm{R}$ employs a multicomponent approach to ensure NMDAR functional enhancement while limiting toxicity-inducible elements may represent a fruitful avenue of further investigation, specifically for gaining further insights into the structural (which domains/sequences of proteins) and time-dependent (acute vs. chronic) aspects of the $\sigma$-1R-mediated disruption of targeted protein-protein interactions. Further studies are also needed to determine if $\sigma-1 \mathrm{R}$ interaction with NMDAR alone plays a critical role in the $\sigma-1 \mathrm{R}$ mediated increase in NMDAR function. Delving deeper into these alluring aspects $-\sigma-1 \mathrm{R}$-mediated, targeted disruption of interaction between NMDAR and nNOS - using $\sigma-1 \mathrm{R}$ knockout mice will validate profiling of $\sigma-1 \mathrm{Rs}$ as a neuroprotective target.

In conclusion, obtaining in-depth mechanistic insights into $\sigma-1 \mathrm{R}$ modulation of NMDAR function that altogether preserves $\mathrm{Ca}^{2+}$ homoeostasis would provide valuable information for designing novel as well as improving prevailing therapeutic strategies. 


\section{References}

1 Choi DW, Maulucci-Gedde M, Kriegstein AR: Glutamate neurotoxicity in cortical cell culture. J Neurosci 1987;7:357-368.

2 Bliss TV, Lomo T: Long-lasting potentiation of synaptic transmission in the dentate area of the anaesthetized rabbit following stimulation of the perforant path. J Physiol 1973;232: 331-356.

3 Dunwiddie TV, Lynch G: The relationship between extracellular calcium concentrations and the induction of hippocampal long-term potentiation. Brain Res 1979;169:103-110.

4 Hardingham GE, Bading $\mathrm{H}$ : The Yin and Yang of NMDA receptor signalling. Trends Neurosci 2003;26:81-89.

5 Hardingham GE, Fukunaga Y, Bading H: Extrasynaptic NMDARs oppose synaptic NMDARs by triggering CREB shut-off and cell death pathways. Nat Neurosci 2002;5. 405-414.

6 Paoletti P, Bellone C, Zhou Q: NMDA receptor subunit diversity: impact on receptor properties, synaptic plasticity and disease. Nat Rev Neurosci 2013;14:383-400.

7 Lau CG, Zukin RS: NMDA receptor trafficking in synaptic plasticity and neuropsychiatric disorders. Nat Rev Neurosci 2007;8:413-426.

$8 \mathrm{Su}$ TP, et al: The sigma-1 receptor chaperone as an inter-organelle signaling modulator. Trends Pharmacol Sci 2010;31:557-566.

9 Hayashi T, Su TP: Sigma-1 receptor chaperones at the ER-mitochondrion interface regulate $\mathrm{Ca}^{2+}$ signaling and cell survival. Cell 2007; 131:596-610.

10 Kourrich S, et al: The sigma-1 receptor: roles in neuronal plasticity and disease. Trends Neurosci 2012;35:762-771.

11 Martina M, et al: The sigma-1 receptor modulates NMDA receptor synaptic transmission and plasticity via SK channels in rat hippocampus. J Physiol 2007;578(pt 1):143-157.
12 Bergeron R, de Montigny C, Debonnel G: Bi phasic effects of sigma ligands on the neuronal response to N-methyl-D-aspartate. Naunyn Schmiedebergs Arch Pharmacol 1995; 351:252-260.

13 Liang X, Wang RY: Biphasic modulatory action of the selective sigma receptor ligand SR 31742A on N-methyl-D-aspartate-induced neuronal responses in the frontal cortex. Brain Res 1998;807:208-213.

14 Maurice T, Privat A: SA4503, a novel cognitive enhancer with sigmal receptor agonist properties, facilitates NMDA receptor-dependent learning in mice. Eur J Pharmacol 1997;328:9-18.

15 Maurice T, et al: PRE-084, a sigma selective PCP derivative, attenuates MK-801-induced impairment of learning in mice. Pharmacol Biochem Behav 1994;49:859-869.

16 Fletcher EJ, et al: Blockade by sigma site ligands of $\mathrm{N}$-methyl-D-aspartate-evoked responses in rat and mouse cultured hippocampal pyramidal neurones. Br J Pharmacol 1995; 116:2791-2800

17 Balasuriya D, Stewart AP, Edwardson JM: The sigma-1 receptor interacts directly with GluN1 but not GluN2A in the GluN1/ GluN2A NMDA receptor. J Neurosci 2013; 33:18219-18224.

18 Bergeron R, de Montigny C, Debonnel G: Potentiation of neuronal NMDA response induced by dehydroepiandrosterone and its suppression by progesterone: effects mediated via sigma receptors. J Neurosci 1996;16: 1193-1202.

19 Li Z, et al: Dehydroepiandrosterone sulfate prevents ischemia-induced impairment of long-term potentiation in rat hippocampal CA1 by up-regulating tyrosine phosphorylation of NMDA receptor. Neuropharmacology 2006;51:958-966.

20 Yoon SY, et al: An increase in spinal dehydroepiandrosterone sulfate (DHEAS) enhances NMDA-induced pain via phosphorylation of the NR1 subunit in mice: involvement of the sigma-1 receptor. Neuropharmacology 2010; 59:460-467.
21 Kim HW, et al: Activation of the spinal sigma-1 receptor enhances NMDA-induced pain via PKC- and PKA-dependent phosphorylation of the NR1 subunit in mice. Br J Pharmacol 2008; 154:1125-1134.

22 Pabba M, et al: NMDA receptors are upregulated and trafficked to the plasma membrane after sigma-1 receptor activation in the rat hippocampus. J Neurosci 2014;34:1132511338.

23 Sanchez-Blazquez P, et al: The calcium-sensitive sigma-1 receptor prevents cannabinoids from provoking glutamate NMDA receptor hypofunction: implications in antinociception and psychotic diseases. Int J Neuropsychopharmacol 2014;1-13.

24 Zhang Y, et al: Sigma-1 receptor agonists provide neuroprotection against gp120 via a change in bcl-2 expression in mouse neuronal cultures. Brain Res 2012;1431:13-22.

25 Goyagi T, et al: Neuroprotective effect of $\sigma_{1}$ receptor ligand 4-phenyl-1-(4-phenylbutyl) piperidine (PPBP) is linked to reduced neuronal nitric oxide production. Stroke 2001;32: 1613-1620.

26 Yang ZJ, et al: Sigma receptor ligand 4-phenyl-1-(4-phenylbutyl)-piperidine modulates neuronal nitric oxide synthase/postsynaptic density-95 coupling mechanisms and protects against neonatal ischemic degeneration of striatal neurons. Exp Neurol 2010;221:166174.

27 Aarts M, et al: Treatment of ischemic brain damage by perturbing NMDA receptorPSD-95 protein interactions. Science 2002; 298:846-850

28 Nedergaard M, Verkhratsky A: Calcium dyshomeostasis and pathological calcium signalling in neurological diseases. Cell Calcium 2010;47:101-102. 